\title{
PROGRAMA DE VACUNACIÓN CONTRA Metapneumovirus aviar EN POLLOS DE CARNE
}

\author{
Vaccination Program Against A vian metapneumovirus in Broilers
}

Rosa Ríos H. ${ }^{1}$, Eliana Icochea D'A. ${ }^{1,2}$, Pablo Reyna S. ${ }^{1}$, Rosa Gonzáles V. ${ }^{1}$, Mercedes Sialer G. ${ }^{3}$, Néstor Falcón $\mathrm{P}_{.}{ }^{4,5}$ y Alexander Torre Ch. ${ }^{1}$

\section{Resumen}

Se evaluó la efectividad de un programa de vacunación contra Metapneumovirus aviar (aMPV) en pollos de carne, empleando una vacuna a virus vivo contra el aMPV conteniendo la cepa pollo 1062 subtipo $\mathrm{B}$, con un título de $10^{2.4} \mathrm{DICT}_{50}$. La vacuna se administró al $1^{\text {er }}$ día de edad por aspersión. El estudio se realizó en dos granjas comerciales de una integración avícola ubicada en una zona donde el Síndrome de Cabeza Hinchada $(\mathrm{SCH})$ es un problema frecuente. Se utilizó un total de 185593 pollos de 1 día de edad, de ambos sexos, de la línea Cobb Vantress, distribuidos en un grupo experimental vacunado contra el aMPV y un grupo control no vacunado contra aMPV. Se evaluó la presentación de $\mathrm{SCH}$, intensidad de signos clínicos respiratorios, lesiones post mortem, parámetros productivos, mortalidad y respuesta serológica mediante la prueba de ELISA indirecta. Sólo el grupo experimental presentó aves con SCH durante la $3^{\mathrm{a}}$ y $4^{\mathrm{a}}$ semana de edad, presentando signos clínicos respiratorios más intensos y lesiones post mortem más severas a nivel del tracto respiratorio. El grupo control presentó mejores parámetros productivos (106 y $75 \mathrm{~g}$ adicionales de peso corporal en machos y hembras, $\mathrm{p}<0.05$ ) y 75 y 4 puntos menos de I.C.A. en machos y hembras, respectivamente, que el grupo experimental; sin embargo, presentó una mayor mortalidad acumulada $(0.64 \%)$ que el grupo experimental $(0.42 \%)$ debido a una mayor frecuencia de problemas congestivos. Los resultados sugieren que la vacunación contra aMPV predispone, principalmente a los machos, a la presentación del SCH.

Palabras clave: Metapneumovirus aviar, síndrome de cabeza hinchada, $\mathrm{SCH}$, vacunación, pollos de carne, mortalidad, parámetros productivos

\footnotetext{
${ }^{1}$ Laboratorio de Patología Aviar, ${ }^{4}$ Laboratorio de Medicina Veterinaria Preventiva, Facultad de Medicina Veterinaria, Universidad Nacional Mayor de San Marcos, Lima

${ }^{2}$ E-mail: eliana.icochea@gmail.com

${ }^{3}$ Empresa avícola San Fernando S.A., Lima

${ }^{5}$ Dirección actual: Facultad de Veterinaria y Zootecnia, Universidad Peruana Cayetano Heredia, Lima
} 
A vaccination programme against Avian metapneumovirus (aMPV) in broilers was evaluated using a live virus vaccine against aMPV with the strain chicken 1062, title of $10^{2.4} \mathrm{DICT}_{50}$ sprayed at first day of age. The study conducted in two commercial farms of poultry growers, located in a region where the Swollen Head Syndrome (SHS) is prevalent. A flock of 185593 1-day old broilers of both sexes, Cobb Vantress 500 line, were distributed in a vaccinated group against aMPV and a control group not vaccinated against aMPV. It was evaluated the presentation of SHS, intensity of respiratory signs, post mortem lesions, productive parameters, mortality, and serological response by indirect ELISA test. Only the vaccinated group had birds with SHS during the $3^{\text {rd }}$ and $4^{\text {th }}$ week of age, showing intense respiratory signs and severe post mortem lesions at respiratory tract level. The control group showed better performance than the vaccinated group (additional 106 and $75 \mathrm{~g}$ of body weight in males and females than controls, $\mathrm{p}<0.05$ ), and 75 and 4 points less of conversion index in male and females than controls respectively. However, the control group had a higher accumulative mortality $(0.64 \%)$ than the vaccinated group $(0.42 \%)$, mainly due to congestive problems. The results suggested that in vaccination against aMPV in chicken broilers, males are more sensitive than females to the occurrence of SHS.

Key words: Avian metapneumovirus, Swollen Head Syndrome, SHS, vaccination, broilers, mortality, productive parameters

\section{INTRODUCCIÓN}

El Metapneumovirus aviar (aMPV) es el agente causal de una de las enfermedades respiratorias de importancia en la crianza de aves (Gough, 2003), ocasionando continuas pérdidas económicas debido al incremento de morbilidad y mortalidad, gastos en medicación, y disminución en la producción de huevos en el caso de ponedoras (Hafez, 2005). Esta enfermedad aparece por primera vez en 1979 en Sudáfrica, como un proceso respiratorio que afectó inicialmente a pavos, siendo denominado TRT por las siglas en inglés (Turkey Rhinotracheitis) (Buys y Du Preez, 1980; Giambrone, 1997). Posteriormente, en 1980, la enfermedad fue observada en pollos de 3 a 4 semanas de edad, en donde además de un proceso respiratorio agudo, se caracterizó por presentar celulitis facial, alta morbilidad y rápida diseminación, llamándosele Síndrome de Cabeza Hinchada (SCH) (Morley y Tomson, 1984; Buys et al., 1989).
Los primeros reportes fueron atribuidos a una infección mixta por Echerichia coli y un coronavirus (Morley y Thomson, 1984; Droual y Woolcock, 1994a); sin embargo, en la actualidad, se considera que el aMPV en pollos de carne solo causa una infección respiratoria leve y, únicamente, en complicaciones con infecciones bacterianas secundarias o condiciones medioambientales adversas se presenta el SCH (Giambrone, 1997).

En el Perú, se observó, en 1986, los primeros casos de $\mathrm{SCH}$ en pollos de carne, lo cual coincidió con la importación de pollos beneficiados procedentes de Francia. El aMPV se aisló e identificó por primera vez en 1995 en el país (Rivera et al., 1999; Icochea, 2002), constituyendo desde entonces en uno de los principales problemas de la industria de pollos de carne. El impacto que ocasiona la infección depende de su interacción con otros factores, principalmente condiciones medioambientales como fluctuaciones térmicas, por lo que la enfermedad del SCH es endémica en el verano (Icochea, 2002). 
La vacunación se realiza solo en reproductoras para prevenir las caídas de producción; sin embargo, se requiere evaluar si la vacunación en pollos de carne es efectiva en prevenir los signos y lesiones respiratorios que ocasiona el SCH. Por ello, el presente estudio tuvo como objetivo evaluar la efectividad de un programa de vacunación contra aMPV en pollos de carne utilizando una vacuna a virus vivo contra el aMPV aplicada al primer día de edad.

\section{Materiales y Métodos}

\section{Lugar de Ejecución}

El presente trabajo se realizó en dos granjas comerciales pertenecientes a una integración avícola dedicada a la producción de pollos de carne, ubicada en el km 98 y 115 de la carretera a Chancay-Huacho, provincia de Chancay, Lima. La evaluación serológica y el análisis de resultados se realizó en el Laboratorio de Patología Aviar de la Facultad de Medicina Veterinaria, Universidad Nacional Mayor de San Marcos, Lima.

\section{Aves}

Se usó un total de 185593 pollos de carne de un día de edad de la línea Cobb-Vantress 500 de ambos sexos, provenientes de dos lotes comeciales de reproductoras jóvenes.

El estudio comprendió dos grupos: un grupo experimental de 46800 machos y 47 112 hembras vacunadas al día de edad contra aMPV, y un grupo control de aves no vacunadas contra aMPV que contó con 45000 machos y 46681 hembras. Ambos grupos fueron criados en granjas separadas a una distancia de $17 \mathrm{~km}$ entren sí, bajo similares condiciones de manejo, infraestructura de galpones y equipos de crianza.

\section{Vacuna \\ La vacuna fue a virus vivo contra Meta- pneumovirus aviar (aMPV) conteniendo la}

cepa pollo 1062 subtipo $\mathrm{B}$, con un título de $10^{2.4} \mathrm{DICT}_{50}$ Se administró vía aspersión al primer día de edad en la planta de incubación. Las vacunas respiratorias de ENC y BI fueron administradas en el grupo experimental a los 9 días de edad vía agua de bebida y revacunadas a los 19 días con ENC vía agua de bebida. En el grupo control se aplicó BI al primer día de edad vía aspersión y luego ENC a los 10 días de edad vía aspersión.

\section{Toma de Muestras}

Se colectó 40 muestras de sangre por sexo y grupo a los 1, 21, 28, 35 y 42 días de edad. La titulación de anticuerpos contra el aMPV se hizo mediante la prueba de ELISA indirecta, usando un kit comercial (Laboratorios IDEXX).

\section{Evaluación del Estudio}

Se evaluó la presentación del SCH y signos clínicos respiratorios (estornudos/min), las lesiones post mortem (en las aves que murieron), parámetros productivos (ganancia de peso vivo, eficiencia en la conversión de alimento) y la tasa de mortalidad. Se empleó la prueba de T de Student para determinar diferencias estadísticas entre los pesos finales de los dos grupos experimentales.

\section{Resultados y Discusión}

Las aves del grupo experimental vacunado presentaron cuadros de $\mathrm{SCH}$ en 70 $(0.15 \%)$ machos y $35(0.07 \%)$ hembras entre la $4^{\mathrm{a}}$ y $5^{\mathrm{a}}$ semana de edad. De ellos, 32 machos murieron por complicaciones por Escherichia coli, en tanto que el resto de machos y todas las hembras se recuperaron.

Infecciones experimentales con el virus no han conseguido reproducir la enfermedad (Turpin et al., 2002; Cook, 2003; Jones, 2004). Sin embargo, Giambrone (1997) señala que infecciones experimentales con 
Cuadro 1. Lesiones respiratorias y congestivas halladas a la necropsia en pollos de carne a partir de la tercera semana de aplicado la vacuna a virus vivo contra Metapneumovirus aviar (aMPV)

\begin{tabular}{|c|c|c|c|c|c|c|c|c|c|}
\hline \multirow{3}{*}{ Tipo de lesión } & \multirow{3}{*}{$\operatorname{Sexo}^{1}$} & \multicolumn{8}{|c|}{ Edad (semanas) } \\
\hline & & \multicolumn{4}{|c|}{ Grupo experimental } & \multicolumn{4}{|c|}{ Grupo control } \\
\hline & & 3 & 4 & 5 & 6 & 3 & 4 & 5 & 6 \\
\hline \multirow{2}{*}{$\begin{array}{l}\text { Mucosidad en cornetes } \\
\text { nasales }\end{array}$} & M & + & & & & + & & & \\
\hline & $\mathrm{H}$ & + & & & & + & & & \\
\hline \multirow{2}{*}{ Congestión traqueal } & M & ++ & + & & + & + & & & + \\
\hline & $\mathrm{H}$ & + & + & & & + & & & \\
\hline \multirow{2}{*}{ Turbidez de sacos aéreos } & M & + & & & & + & + & & \\
\hline & $\mathrm{H}$ & + & & & & + & & & \\
\hline \multirow{2}{*}{ Espuma en sacos aéreos } & M & & + & + & + & & + & + & + \\
\hline & $\mathrm{H}$ & & + & + & + & & + & + & + \\
\hline \multirow{2}{*}{$\begin{array}{l}\text { Material caseoso sacos } \\
\text { aéreos en pleura y pulmón }\end{array}$} & M & & ++ & ++ & & & + & & \\
\hline & $\mathrm{H}$ & & + & & & & & & \\
\hline \multirow{2}{*}{$\begin{array}{l}\text { Exudado caseoso en senos } \\
\text { infraorbitarios }\end{array}$} & M & & ++ & & & & & & \\
\hline & $\mathrm{H}$ & & & & & & & & \\
\hline \multirow{2}{*}{ Hidropericardio y ascitis } & M & & & + & & & & + & \\
\hline & $\mathrm{H}$ & & & + & & & & + & \\
\hline
\end{tabular}

${ }^{1} \mathrm{M}$ : macho; $\mathrm{H}$ : hembra

Lesión ligera $=+$ Lesión moderada $=++$ Lesión severa $=+++$

el aMPV solo causan signos clínicos leves, indicando que se puede obtener hasta un $30 \%$ de cabezas hinchadas dependiendo de las complicaciones bacterianas. Dentro de estas, las más importantes involucran agentes secundarios como E. coli (Bentué, 2005). Además, condiciones medioambientales adversas contribuyen a la presentación del síndrome (Valladares et al., 1996). Estos factores, pueden ayudar a la mayor presentación del SCH en machos, probablemente a que se encuentran asociados a la mayor ganancia de peso corporal, mayor demanda de oxígeno y, por consiguiente, mayor estrés metabólico.
Signos clínicos de tipo respiratorio se observó en ambos grupos desde la $1^{\mathrm{a}}$ a la $5^{\mathrm{a}}$ semana de edad. En el grupo experimental, los signos clínicos fueron más severos, en comparación al grupo control, siendo de moderados a severos entre la $3^{\mathrm{a}}$ y $5^{\mathrm{a}}$ semana de edad, probablemente debido a exposición de campo con el aMPV, asociado a complicaciones post vacunales por los virus de ENC y $\mathrm{BI}$ administrados en el agua de bebida. En el grupo control, las reacciones respiratorias fueron de grado moderado leve entre la $2^{\mathrm{a}} \mathrm{y}$ $4^{\mathrm{a}}$ semana de edad, posiblemente como resultado de una menor exposición de campo y reacción post vacunal contra Bronquitis In- 
Cuadro 2. Rendimiento productivo y mortalidad (\%) a los 42 días de edad, según sexo, en pollos de carne vacunados contra Metapneumovirus aviar (aMPV)

\begin{tabular}{|c|c|c|c|c|c|c|}
\hline Sexo & Grupo & $\begin{array}{l}\text { Peso } \\
(\mathrm{kg})\end{array}$ & $\begin{array}{c}\text { Consumo } \\
\text { alimento } \\
(\mathrm{kg})\end{array}$ & I.C.A. ${ }^{1}$ & I.E.P. ${ }^{2}$ & $\begin{array}{c}\text { Mortalidad } \\
(\%)\end{array}$ \\
\hline \multirow{2}{*}{ Macho } & Experimental & 2.454 & 4.272 & 1.741 & 320.6 & 4.46 \\
\hline & Control & 2.560 & 4.264 & 1.666 & 347.2 & 5.10 \\
\hline \multirow{2}{*}{ Hembra } & Experimental & 2.095 & 3.887 & 1.855 & 273.6 & 2.03 \\
\hline & Control & 2.170 & 3.745 & 1.726 & 281.4 & 2.45 \\
\hline
\end{tabular}

fecciosa y Newcastle, aplicadas vía aspersión en planta de incubación al primer día y en granja a los 10 días, respectivamente. Hay reportes que señalan que los signos clínicos se producen entre las 4 a 6 semanas de edad y tienen una duración dos a tres semanas (Arns y Hafez, 1992). En los casos sin complicaciones secundarias, la recuperación puede ocurrir entre los 10 a 14 días post infección (Giambrone, 1997). Las diferencias entre los programas vacunales de los grupos puede haber influido en la presentación de los signos respiratorios; la vacunaciones en el agua de bebida producen generalmente una menor uniformidad de la respuesta post vacunal causando reacciones post vacunales severas en comparación a la vacunación vía spray. Asimismo, las edades de vacunación influyen directamente sobre la intensidad de las reacciones post vacunales, donde la severidad se incrementa con la edad de las aves; siendo un factor predisponente para el grupo experimental donde se exacerbaron los cuadros clínicos observados por el reto de campo de aMPV en comparación al grupo control.

Lesiones en aves muertas en la $3^{\mathrm{a}}$ semana de edad consistieron en mucosidad en cornetes nasales, congestión traqueal y sacos aéreos turbios. Las lesiones se intensifi- caron entre la $4^{\mathrm{a}}$ y $5^{\mathrm{a}}$ semana de edad en los machos de ambos grupos, aunque sólo las del grupo experimental presentaron exudado caseoso en senos infraorbitarios. A partir de la $5^{\text {a }}$ semana se observaron lesiones de tipo congestivo (ascitis e hidropericardio) en ambos grupos, pero especialmente en el grupo control, probablemente debido a su mayor peso corporal. Las lesiones disminuyeron en ambos grupos en la $6^{\mathrm{a}}$ semana, observándose congestión, mucosidad traqueal y espuma en sacos aéreos en algunas aves (Cuadro 1). El aMPV produce lesiones a nivel del tracto respiratorio superior por un corto tiempo mientras ocurre el proceso de replicación viral (Cook et al., 1991; De Zande et al., 1999), en tanto que se producen lesiones a nivel del tracto respiratorio inferior; además, las lesiones típicas de $\mathrm{SCH}$ al presentarse infecciones secundarias (Droual y woolcock, 1994b; Giambrone, 1997; Icochea, 2002; Gough, 2003), pudiéndose encontrar, también, rinitis, y congestión e inflamación de traquea (Arns y Hafez, 1992; Giambrone, 1997).

En el grupo control se observó un mejor rendimiento productivo, tanto en machos como hembras, que el grupo experimental (Cuadro 2). Así, en el control, los machos ganaron $106 \mathrm{~g}(4.14 \%)$ más de peso vivo, con $4.3 \%$ mejor conversión alimenticia, y $7.6 \%$ 


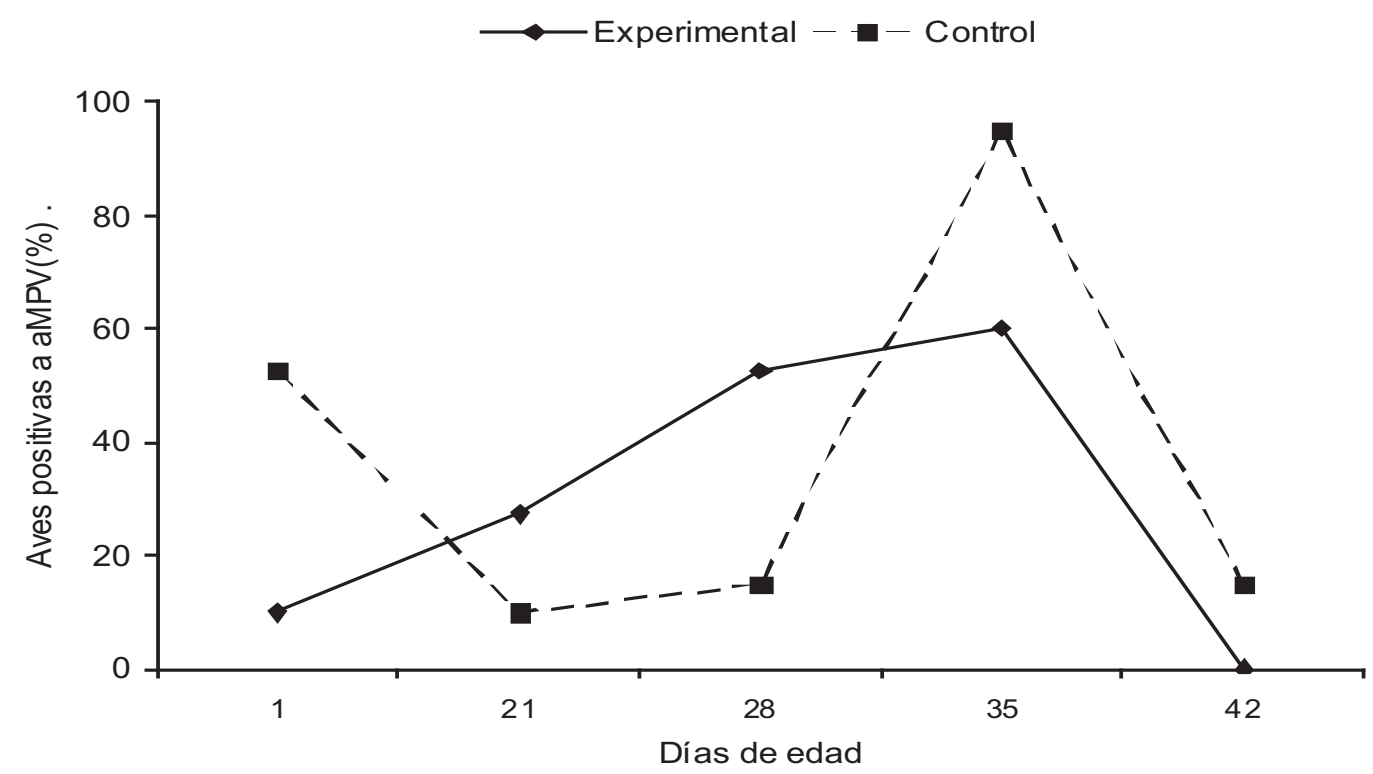

Figura 1. Porcentaje de pollos de carne positivos al aMPV a los 42 días de edad, detectado mediante la prueba de ELISA indirecta, en aves vacunadas contra Metapneumovirus aviar (aMPV) y en aves sin vacunar

mejor índice de eficiencia productiva que los machos del grupo vacunado; similar ventaja presentaron las hembras del grupo control, ganando $75 \mathrm{~g}(3.5 \%)$ adicionales de peso corporal, $6.9 \%$ más eficiente en la conversión de alimento y $2.8 \%$ más de índice productivo que las hembras del grupo experimental, encontrándose diferencias significativas $(p<0.05)$ en ambos sexos.

Existe la posibilidad de una interferencia del virus vacunal de la IBV aplicado a los pollos al día de edad. Sin embargo, el grupo control presentó un mayor porcentaje de mortalidad acumulada en ambos sexos (Cuadro 2), posiblemente debido al mejor desempeño productivo de este grupo, ya que la mortalidad se debió principalmente a problemas congestivos (hidropericardio y ascitis). Giambrone (1997) y Vélez (2005) señalan que las infecciones producidas por aMPV pueden llegar a disminuir los parámetros productivos aumentando los costos de producción, pero es importante destacar que los estudios reportados sobre programas de vacunación consideran aspectos clínicos respiratorios pero no parámetros productivos.

El 10.0 y $52.5 \%$ de las aves fueron positivas a aMPV en el primer día de edad en los grupos experimental y control, respectivamente (Fig. 1), posiblemente debido a las diferencias de respuesta inmunológica entre lotes de aves. Giambrone (1997) señala que los títulos de anticuerpos maternales en pollos de carne de 1 día de edad están directamente relacionados con el nivel de anticuerpos de las reproductoras $y$, según recientes investigaciones, los anticuerpos maternales persisten hasta las 2 a 3 semanas de edad (Hafez, 1994). El mayor nivel de aves positivas al aMPV se presentó a los 35 días de edad (Fig. 1), resultado que demuestra exposición al virus de aMPV de campo en ambos grupos. El perfil serológico demuestra que la respuesta de anticuerpos, obtenida por vacunación o por exposición natural, es de corta duración, y esto se puede deber a que el virus tiene un corto periodo de incubación (Icochea, 2002; Jones, 2004). 


\section{Conclusiones}

Los resultados sugieren que la vacunación contra Metapneumovirus aviar en pollos de carne, usando una vacuna a virus vivo contra el aMPV conteniendo la cepa pollo 1062 subtipo $\mathrm{B}$, con un título de $10^{2.4} \mathrm{DICT}_{50}$, administrado al $1^{\text {er }}$ día de edad por aspersión en la planta de incubación, predispone a la presentación de síndrome de cabeza hinchada en pollos de carne.

\section{Literatura Citada}

1. Arns C, Hafez, H. 1992. Swollen Head Syndrome in poultry flocks in Brazil. Proc LII Western Poultry Disease Conference. Sacramento, California. $\mathrm{p}$ 81-83.

2. Bentué M. 2005. El virus del TRT afecta ponedoras comerciales. Mundo Avícola y Porcino 3: 3-5.

3. Buys SB, Du Preez JH. 1980. A preliminary report on the isolation of a virus causing sinusitis in turkeys in South Africa and attempts to attenuate the virus Turkeys. Virus Res 11: 28-36.

4. Buys SB, Du Preez JH, Els HJ. 1989. Swollen Head Syndrome in chickens: a preliminary report on the isolation of a possible etiological agent. J S Afr Vet Assoc 60: 221-222.

5. Cook JKA, Ellis M, Huggins M. 1991. The pathogenesis of turkey rhinotracheitis virus in turkey poults inoculated with the virus alone or together with two strains of bacteria. Avian Pathol 20: 155-166.

6. Cook JKA, Huggins MB, Orbell SJ, Mockett AP. 1995. Protection provided by a commercially available vaccine against different strains of turkey rhinotracheitis virus. Vet Rec 136: 392-393.

7. Cook J. 2003. Síndrome de Cabeza Hinchada: una evaluación. En: Memorias XVIII Congreso Latinoamericano de Avicultura. Santa Cruz, Bolivia.
8. De Zande VS, Nauwynck K, Jonghe S, Pensaert M. 1999. Comparative pathogenesis of a subtype A with a subtype B avian pneumovirus in turkeys. Avian Pathol 28: 239-244.

9. Droual R, Woolcock P. 1994a. Swollen Head Syndrome associated with E. coli and infection bronquitis virus in the central valley of California. Avian Pathol 23: 733-742.

10. Droual R, Woolcock P. 1994b. Swollen Head Syndrome in broiler chickens: a reevaluation of viral etiology. In: Proc XLII Western Poultry Disease Conference. Sacramento, California.

11. Giambrone J. 1997. Síndrome de Cabeza Hinchada. Avicultura Profesional 15(5/6): 33-36.

12. Gough RE. 2003. Avian Pneumoviruses. In: Diseases of poultry. Calnek BW, Barnes HJ, Beard CW (eds). 11 $1^{\text {th }}$ ed. Arnes, USA: Iowa University Press. p 92-99.

13. Hafez H. 1994. Current situation on turkey rhinotracheitis and Swollen Head Syndrome. Proc XLIII Western Poultry Disease Conference. Sacramento, California 27(1): 91-93.

14. Hafez H. 2005. Doenças respiratorias em perus. En: Conferencia APINCO de Ciencia e Tecnología Avícolas. Palestras do Temario Peral. Simpósio de Perus. São Paulo, Brasil. Vol II: 269-284.

15. Icochea E. 2002. Infecciones por pneumovirus aviar. En: I Seminario Internacional de Avicultura. Lima, Perú: AMEVEA.

16. Jones $\boldsymbol{R C}$. 2004. Importancia de las infecciones a pneumovirus aviar en los procesos respiratorios. En: II seminario Internacional AMEVEA. Avicultura con visión global. Lima, Perú: AMEVEA.

17. Morley A, Thomson D. 1984. Swollen Head Syndrome in broiler chickens. Avian Dis 28: 238-243.

18. Rivera H, Icochea E, Hung A, Ramírez $A$, Rosadio R. 1999. Etiología del Síndrome de Cabeza Hinchada en broilers. En: XVI Congreso Latinoamericano de Avicultura. Lima, Perú. p 271. 
19. Turpin E, Perkins L, Swayne D. 2002. Experimental infection of turkeys with avian pneumovirus and either Newcastle disease virus or Escherichia coli. Avian Dis 46: 412-422.

20. Valladares J, Barrientos L, Angulo E, Juárez D. 1996. Detección serológica del virus de rinotraqueítis del pavo y su probable relación con enfermedad respiratoria en el noreste de México. En:
Memorias XXI Convención Anual. Cancún, México: Asociación Nacional de Especialistas en Ciencias Avícolas. p 338-340.

21. Vélez S. 2005. Enfermedades respiratorias: interacción de pneumovirus aviar y micoplasma aviar en el campo y sus alternativas para su control. En: Jornada Técnica Fort Dodge América Latina. Lima, Perú. 